\title{
26 CURRENT EVIDENCE ON PUBLICISED SOBRIETY CHECKPOINT PROGRAMMES: ARE THEY STILL EFFECTIVE?
}

doi:10.1136/injuryprev-2012-040580d.26

G Bergen* ${ }^{*}$ A Pitan, RA Shults, S Ou, DA Sleet. Centers for Disease Control and Prevention, 4770 Buford Highway, Atlanta, Georgia, USA

Background Alcohol-impaired driving is a major contributor to motor vehicle crash deaths resulting in almost 270000 deaths globally. In 2001, the Community Preventive Services Task Force recommended the use of sobriety checkpoints on the basis of a systematic review that showed sobriety checkpoints resulted in a median decline of about $20 \%$ in alcohol-related crash fatalities. This review included 32 studies published through 30 June 2000. New studies evaluating publicised sobriety Checkpoint programmes have since been published, leading to an updated review in 2012.

Aims/Objectives/Purpose To determine if the effectiveness of sobriety checkpoints has changed since the publication of the 2001 (original) systematic review.

To explore whether low manpower staffed checkpoints are as effective as traditional checkpoints.

To determine the economic costs and benefits of sobriety checkpoints. 
Methods The Community Guide methods for systematic reviews were used to evaluate studies of sobriety checkpoints published from July 2000 through March 2012.

Results/Outcomes Fifteen studies met the systematic review inclusion criteria. Fourteen studies conducted in the USA showed a median reduction in alcohol-involved fatal crashes of $8.9 \%$. One study from New Zealand showed a reduction in serious and fatal nighttime crashes of $22 \%$. The two evaluations on low manpower staffed checkpoints respectively showed reductions of $18 \%$ and $64 \%$ in alcohol-involved fatal crashes and drinking/ driving behaviour. Five economic evaluations showed that benefits exceed costs with benefit-to-cost ratios ranging from $2: 1$ to $57: 1$.

Significance/Contribution to the Field This systematic review update confirms the effectiveness of sobriety checkpoints in reducing alcohol-impaired crashes, injuries and deaths. 